\title{
A connection-level call admission control using genetic algorithm for multi-class multimedia services in wireless networks
}

\author{
Xiao Hong \\ Computer Center \\ Changchun Institute of Technology \\ TONGZHI Street 80\#, Changchun \\ Jilin Province, 130021, P.R. China \\ E-mail: xiaohongpc@tom.com
}

\author{
Yang Xiao* \\ Department of Computer Science \\ The University of Memphis \\ Memphis, TN 38152, USA \\ E-mail: yangxiao@ieee.org \\ *Corresponding author
}

\section{Qiang Ni and Tianji Li}

The Hamilton Institute

National University of Ireland

Maynooth, Ireland

E-mail: Qiang.Ni@ieee.org

E-mail: Tianji.Li@nuim.ie

\begin{abstract}
Semi-Markov Decision Process (SMDP) can be used to optimise channel utilisation with upper bounds on handoff blocking probabilities as Quality of Service constraints for call admission control in a wireless cell in a Personal Communication System (PCS). However, this method is too time consuming and therefore it fails when state space and action space are large. In this paper, we apply a genetic algorithm approach to address the situation when the SMDP approach fails. We code call admission control decisions as binary strings. The coded binary strings are feed into the genetic algorithm, and the resulting binary strings are founded to be near optimal call admission control decisions. Simulation results from the genetic algorithm are compared with the optimal solutions obtained from linear programming for the SMDP approach. The results reveal that the genetic algorithm approximates the optimal approach very well with less complexity.
\end{abstract}

Keywords: call admission control; multimedia; Quality of Service (QoS; queuing system; genetic algorithm; Semi-Markov Decision Process (SMDP); wireless/mobile networks.

Reference to this paper should be made as follows: Hong, X., Xiao, Y., Ni, Q. and $\mathrm{Li}, \mathrm{T}$. (2006) 'A connection-level call admission control using genetic algorithm for multi-class multimedia services in wireless networks', Int. J. Mobile Communications, Vol. 4, No. 5, pp.568-580. 
Biographical notes: Xiao Hong is Associate Professor of Computer Center at Changchun Institute of Technology, Changchun, China. She teaches all kinds of computer science courses.

Yang Xiao is an IEEE Senior member. He was a voting member of IEEE 802.11 Working Group from 2001 to 2004. He currently serves as Editorin-Chief for International Journal of Security and Networks and for International Journal of Sensor Networks. He serves as Associate Editor or on editorial boards for the following journals: (Wiley) International Journal of Communication Systems, (Wiley) Wireless Communications and Mobile Computing, EURASIP Journal on Wireless Communications and Networking, and International Journal of Wireless and Mobile Computing. He serves as guest editor for six journal special issues. He serves as a technical programme Vice-Chair for two conferences, a symposium Co-Chair for two conferences, a coeditor for five edited books, a TPC member for more than 50 conferences/symposia/workshops, and a reviewer for many journals, conferences and funding agencies. He has served as a panelist for NSF. His research areas are wireless networks, mobile computing, and network security.

Qiang Ni is currently a Senior Researcher at the Hamilton Institute, National University of Ireland, Maynooth. He received the BS, MS and PhD degrees from Hua Zhong University of Science and Technology (HUST), China in 1993, 1996 and 1999 respectively. From 1999 to 2001, he was a post-doctoral research fellow in HUST. He visited Microsoft Research Asia Lab during the year of 2000. In 2001, he joined INRIA, France, where he was a researcher at the Planète group. Since 2002, he has been active as a voting member for the IEEE 802.11 wireless LAN Standard Working Group. He has served as TPC for the IEEE Globecom 05, WirelessCom 2005, IEEE VTC 2003, IEEE Sarnoff 2005, and IEEE WiOPT'05. His current research interests include wireless communication network protocol design and analysis, vertical handover and mobility management in mobile networks, and multimedia transmission over hybrid wired/wireless networks.

Tianji Li received his BS and MS degrees in Computer Science from JiLin and ZhongShan Universities, China, in 1998 and 2001, respectively, and his MS degree in Networking and Distributed Computation from Ecole Doctorale STIC, Universite de Nice-Sophia Antipolis, France, in 2004. Currently, he is working towards a $\mathrm{PhD}$ degree at Hamilton Institute, National University of Ireland at Maynooth, Ireland. From 2001 to 2003, he was a Software Engineer at the Beijing Research Institute of Huawei Technologies, China. His research interests are performance evaluation and optimisation in wireless networks.

\section{Introduction}

The Quality of Service (QoS) guarantee for multimedia services is important in wireless networks. Call Admission Control (CAC) is vital for the QoS guarantee owing to limited capacity in wireless networks. A good CAC needs to efficiently utilise system resources and satisfy QoS requirements. Furthermore, in order to improve the overall performance of the system, sometimes, denying some resource requests even when excess capacity exists is an essential feature for CAC (Yener and Rose, 1997).

Many CAC schemes in wireless/mobile networks have been proposed in literature (Ayyagari and Ephremides, 1999; Ho and Lea, 1999; Ramjee et al., 1997; Kwon et al., 1999; Kwon et al., 1998; Choi et al., 2000; Kwon et al., 1998; Yoon and Lee, 1999; 
Kwon et al., 2003; Biswas and Sengupta, 1997; Yener and Rose, 1997; Rose and Yate, 1996; Xiao et al., 2001; Xiao et al., 2002; Xiao et al., 2005). Some studies focus on optimal CAC schemes for handoff calls and new calls in a non-multimedia situation, in which Factional Guard Channel is the optimal approach (Ho and Lea, 1999; Ramjee et al., 1997). Quite a few studies seek CAC schemes for multi-classes of multimedia services in a non-adaptive multimedia situation (Ayyagari and Ephremides, 1999; Choi et al., 2000; Kwon et al., 1998; Yoon and Lee, 1999), in which Choi et al. (2000) present a centralised optimal CAC using the Semi-Markov Decision Process (SMDP), and Kwon et al. (1998) and Yoon et al. (Yoon and Lee, 1999) propose distributed optimal CAC using the SMDP approach. Most recently, many researches have focused on adaptive multimedia services (Kwon et al., 1999; Kwon et al., 1998; Kwon et al., 2003; Naghshineh and Willebeek-LeMair, 1997; Alwan, 1996; Xiao et al., 2001; Xiao et al., 2002; Xiao et al., 2005). In our previous work (Xiao et al., 2001), we give an optimal solution using the SMDP approach for adaptive multimedia services. In our previous work, Xiao et al. (2002) and Xiao et al. (2005), we propose bandwidth degradation QoS provisioning and proportional degradation services, respectively, for multiple classes of adaptive multimedia. QoS over wireless networks is also studied in Wee and Gutierrez (2005). Overflow replacement policies in location management are studied in Lou et al. (2004). Genetic algorithm is also used to study the performance of TCP over satellite networks in Karthik et al. (2004), and other publications (Wang and Wang, 2005; Biaz and $\mathrm{Ji}, 2005$ ).

In this paper, we assume $m$ classes of users in a cell. The fixed total number of channels in a cell is $C$. We can model resource allocation in a cell as an $M / M / C / C$ queuing system with $m$ classes of users. The SMDP approach can be adopted in CAC to provide an optimal solution to optimise the channel utilisation for service providers while satisfying the QoS requirements for end users, i.e. upper bounds on handoff blocking probabilities. Moreover, linear programming can be used to solve optimal decision problems. However, the SMDP method fails when state space and action space are too large. The action space increases exponentially with $m$, and the state space is extremely large for a large $C$.

In this paper, we apply a genetic algorithm approach to address such problems as when the SMDP approach fails. Genetic algorithm is based on natural selection. It mimics nature by combining genetics and natural selection in a computer to find near-optimal solutions. Genetic algorithms are a class of operators that are used to evolve solutions to extremely complex problems. The problems may be too broad, too expensive or too complex to be algorithmically solved. A genetic algorithm uses operators like crossover and mutation to find a solution, which is evaluated by an objective function to determine its fitness. If it is more fitful, it replaces the original solution in the set of solutions under consideration. Genetic operators may not give the theoretically best solution, but it will give a better one. How good a solution is depends on the problem, iterations and genetic operator parameters (Collier, 2000). In this paper, we will show that our genetic algorithm does give a very good solution in this special application.

The rest of this paper is organised as follows. We describe the problem definition in Section 2. Section 3 gives an overview of the optimal approach (SMDP), its limitation and scenarios when it fails. Section 4 demonstrates our genetic algorithm. We illustrate simulation results in Section 5. Finally, we conclude this paper in Section 6. 


\section{Problem definition}

In Section 2.1, we first describe a traffic model. Then, we address objectives of this paper in Section 2.2.

\subsection{Traffic model}

We assume $m$ classes of users: $\{1,2, \ldots m\}$. A user in class- $i$ requires $b_{i}$ units of bandwidth or channels. We can model a cell with an $M / M / C / C$ queuing system with $m$ classes of users. Arrival events include new call arrival events and handoff call arrival events; service departures include call completions and handoffs to other cells. We consider only a fixed capacity in a cell, i.e. the total number of bandwidth (channels) is $C$. We have the following assumptions:

- Call requests of each class follow a Poisson distribution;

- The call holding time (service time) of each class is exponentially distributed;

- All the arrival distributions and the call holding time distributions are independent of each other.

Let $\lambda_{i, n}$ denote the new call arrival rate for class- $i$ users; let $\lambda_{i, h}$ denote the handoff call arrival rate for class- $i$ users; let $\mu_{i}$ denote the service rate for class- $i$ users; let $h$ denote the rate of handoffs to other cells. We assume that the handoff rate $h$ is independent of classes.

\subsection{Objectives}

Define a system state of a cell as a vector $x=\left\{x_{1}, x_{2}, \ldots, x_{m}\right\}$, where $x_{i}$ denotes the number of active class- $i$ calls in the cell.

Let $P_{i, H B}$ denote handoff blocking probability for class- $i$ users. Let $D_{i, H B}$ denote a predefined upper bound on handoff blocking probability for class- $i$ users. QoS requirements are defined as follows:

$$
P_{i, H B} \leq D_{i, H B} \text { for } i=1,2, \ldots, m \text {. }
$$

We define the channel utilisation as follows:

$$
U=\sum_{i=1}^{m} b_{i} x_{i} .
$$

Our goal is to maximise the channel utilisation $(U)$ for service providers and to satisfy QoS requirements for end users, i.e. upper bounds on handoff blocking probabilities.

\section{Optimal solution overview}

The Semi-Markov Decision Process (SMDP) is a well-known technique for solving optimisation problems (Choi et al., 2000; Kwon et al., 1998; Yoon and Lee, 1999; Xiao et al., 2001). In this section, we first introduce the definition of the SMDP approach in Section 3.1. Then, we summarise its usage to solve optimisation problems in Section 3.2. 
We provide an overview of how to use the SMDP approach in Section 3.3. Finally, in Section 3.4, we address limitations of the SMDP approach.

\subsection{Semi-Markov Decision Process}

The SMDP is a dynamic system with Markovian properties. This dynamic system at random points in time is observed and classified into one of a finite number of states. At the same time, a decision has to be made, and a cost is incurred owing to the decision made (Tijms, 1986). The decision is chosen from a finite decision space that may depend on the current state. Markovian properties mean that if at a decision epoch the action is chosen in the current state, the costs incurred, the time until, and the state at the next decision epoch depend only on the current state and the chosen action, and are thus independent of the past history of the system.

\subsection{Optimal solution framework}

We summarise a framework to get the optimal solution by the SMDP approach. Firstly, a model should satisfy SMDP definitions/properties. Secondly, we need to properly define system states $(\mathbf{x})$, state space $(\mathbf{S})$, decision epochs, actions (a) and action space $(\Lambda)$. Thirdly, we need to formulate Expected Sojourn Time analytically, when it is in the present state $\mathbf{x}$ and the action $\mathbf{a}$ is taken. Fourthly, we need to formulate transition probability analytically, which is the probability that at the next decision epoch the system will be state $\mathbf{y}$ if the present state is $\mathbf{x}$ and the action $\mathbf{a}$ is taken. Fifthly, we need to define a cost function or a reward function. Sixthly, we need to formulate linear programming formulas (Tijms, 1986) with the cost/reward function. Finally, we solve the linear programming formulas. The solution was proved to be optimal mathematically (Tijms, 1986).

\subsection{Optimal solution}

Define a system state of a cell as a vector $\mathbf{x}=\left\{x_{1}, x_{2}, \ldots, x_{m}\right\}$, where $x_{i}$ denotes the number of active class-i calls in the cell. The state space is given by

$$
\mathbf{S}=\left\{\mathbf{x}: x_{i} \geq 0 \text { for } i=1,2, \ldots, m ; \sum_{i=0}^{m} b_{i} x_{i} \leq C\right\}
$$

An action is defined as:

$$
\mathbf{a}=\left(a_{1}, a_{2}, \ldots a_{2 m}\right\}, a_{i} \in\{0,1\} \text { for } i=1,2, \ldots, m .
$$

If $a_{i}=1$, it means acceptance. If $a_{i}=0$, it means rejection. For $i \leq m, a_{i}$ denotes the action for a new call arrival for a class- $i$ user. For $i \geq m+1, a_{i}$ denotes the action for a handoff call arrival for a class- $(i-m)$ user. A call admission control policy is simply a mapping of a system state to an action. The action space is defined by

$$
\Lambda=\left\{\mathbf{a}: a_{i}=0 \text { if } \mathbf{x}+\mathbf{e}_{\mathbf{i}} \notin \mathbf{S}\right\}
$$

where $e_{i}$ is a vector of zeros, except for a ' 1 ' in the $i$-th position. The reward function is the channel utilisation. The Expected Sojourn Time, the transition probability and the linear programming formulas are defined as follows: 
Let $\tau(\mathbf{x}, \mathbf{a})$ denote Expected Sojourn Time if the system in state $\mathbf{x} \in \Lambda$ and an action $\mathbf{a}$ is taken. Let $\operatorname{Pr}(\mathbf{y} \mid(\mathbf{x}, \mathbf{a}))$ denote the transition probability that at the next decision epoch the system will be state $\mathbf{y}$ if the present state is $\mathbf{x}$ and the action $\mathbf{a}$ is taken. Based on Kwon et al. (1998) and Xiao et al. (2001), we have:

$$
\begin{gathered}
\tau(\mathbf{x}, \mathbf{a})=\frac{1}{\sum_{i=0}^{m}\left[\lambda_{i, n} a_{i}+x_{i} \mu_{i}+x_{i} h+\lambda_{i, h} a_{m+i}\right]} \\
\operatorname{Pr}(\mathbf{y} \mid(\mathbf{x}, \mathbf{a}))=\left\{\begin{array}{l}
\left(\lambda_{i, n} a_{i}+\lambda_{i, h} a_{m+i}\right) \tau(\mathbf{x}, \mathbf{a}), \text { if } y=x+e_{i} \\
\left(x_{i} \mu_{i}+x_{i} h\right) \tau(\mathbf{x}, \mathbf{a}), \text { if } y=x-e_{i} \\
0, \text { otherwise }
\end{array} .\right.
\end{gathered}
$$

Utilisation under $(\mathbf{x}, \mathbf{a})$, i.e., if the present state is $\mathbf{x}$ and the action $\mathbf{a}$ is taken, is defined as Equation (2). Therefore, we can define the following linear programming equations (Kwon et al., 1998; Xiao et al., 2001). The linear programming associated with the SMDP for maximum revenue is given below with decision variables $\pi_{\mathbf{x a}}, \mathbf{x} \in \mathrm{S}, \mathbf{a} \in \Lambda_{\mathbf{x}}$ :

$$
\begin{aligned}
& \text { Maximise } \sum_{\mathbf{x} \in S} \sum_{\mathbf{a} \in \wedge_{x}} U \tau(\mathbf{x}, \mathbf{a}) \pi_{\mathbf{x a}} \text { such that: } \\
& \sum_{\mathbf{x} \in S} \sum_{\mathbf{a} \in \wedge_{x}} \tau(\mathbf{x}, \mathbf{a}) \pi_{\mathbf{x a}}=1 \\
& \sum_{\mathbf{a} \in \wedge_{x}} \pi_{\mathbf{y a}}=\sum_{\mathbf{x} \in S} \sum_{\mathbf{a} \in \wedge_{x}} P(\mathbf{y} \mid \mathbf{x}, \mathbf{a}) \pi_{\mathbf{x a}} \text { for } \boldsymbol{y} \in \mathbf{S} \\
& \pi_{\mathbf{x a}} O, \mathbf{x} \in \mathbf{S}, \mathbf{a} \in \Lambda_{\mathbf{x}}, \\
& \sum_{\mathbf{x} \in S} \sum_{\mathbf{a} \in \wedge_{x}}\left(1-a_{2}\right) \tau(\mathbf{x}, \mathbf{a}) \pi_{\mathbf{x a}} \leq D_{i, H B}
\end{aligned}
$$

The term $\tau(\mathbf{x}, \mathbf{a}) \pi_{\mathbf{x a}}$ can be interpreted as the long run fraction of the decision epochs at which the system is in state $\mathbf{x}$, and the action $\mathbf{a}$ is chosen. We consider the QoS parameter's requirement, an upper bound on handoff dropping probability, in the last equation, where $D_{i, H B}$ is the upper bound on handoff dropping probability for class-i. Based on the above linear programming equations, we can obtain optimal CAC decisions by a linear programming algorithm known as the Interior-Point Methods (Zhang, 1995; Zhao, 1999).

\subsection{The limitation of the SMDP approach}

For every possible state, a call admission control policy found by the SMDP approach must be specified by a complete enumeration of the decisions (Rose and Yate, 1996). The SMDP method fails when the state space and the action space are too large. The action space increases exponentially with $m$, and the state space also increases exponentially with $C$. The variable space for the linear programming formulas increases exponentially with $m$ and $C$, and so does the number of the linear programming formulas. In other words, the SMDP approach is not scalable at all. 
However, the SMDP approach has at least theoretical significance, and it is the criteria for other approaches. A good approach should compare itself with the SMDP approach to ensure getting similar performance with less complexity. The objective of this paper is to provide a similar performance to the SMDP approach with much less computational complexity.

\section{Genetic algorithm}

In this paper, we propose a near-optimal solution using genetic algorithm, which is based on natural selection that mimics nature by combining genetics and natural selection on a computer. We apply a genetic algorithm approach to address such problems as when the SMDP approach fails. We first present the coding method for CAC in Section 4.1. Then, we present the algorithm and the implementation remarks in Section 4.2.

\subsection{Coding}

We code the call admission control decisions as a sequence of $2 m$-bit binary strings corresponding to Equation (4). A value of ' 1 ' of the string stands for the decision to accept a call, and a value of ' 0 ' of the string stands for the decision to reject a call. For $i \leq m$, the value in the position $i$ of the string denotes the action for a new call arrival for a class-i user. For $i \geq m+1$, the value in the position $i$ of the string denotes the action for a handoff call arrival for a class- $(i-m)$ user. For example, at a system state, a coding is 0011. Here, $m$ equals 2 and it stands for the number of classes. We can interpret the coding 0011 as follows:

- The first 0 in 0011 stands for the rejection of a new call arrival in class- 1 . In other words, if the next call request is a new call and it is from class- 1 , CAC will reject it.

- Similarly, the second 0 in 0011 stands for the rejection of a new call arrival in class-2. In other words, if the next call request is a new call and it is from class-2, CAC will reject it.

- The first 1 in 0011 stands for the acceptance of a handoff call arrival in class- 1 . In other words, if the next call request is a handoff call and it is from class-1, CAC will accept it.

- Similarly, the second 1 in 0011 stands for the acceptance of a handoff call arrival in class-2. In other words, if the next call request is a handoff call and it is from class-2, CAC will accept it.

- Each system state is associated with such a string. In other words, for each system state, we need to find a decision coding string. The call admission control decision space includes all the possible $2 m$-length binary strings. Each such string stands for a unique call admission control decision. The size of the space is $2^{2 m}$.

Note that this decision space is different from the action space, which is used in the SMDP approach, and is constrained. However, the call admission control decision space is unconstrained. We do allow accepting a call even though there is no available bandwidth. If this happens, the call will be blocked eventually. 
The resulting binary strings from the genetic algorithm are the near-optimal call admission control decisions.

\subsection{Genetic algorithm}

The idea is to use simple genetic operators to optimise by natural selection. Similarly to natural evolution, the more the chromosome fits, the more likely its genes will be propagated through subsequent generations. The algorithm is described in the following steps.

\section{Step 1}

We first randomly choose an initial set population of strings among $2^{2 \mathrm{~m}}$ strings of decision space. We call an individual decision string a chromosome. A string is composed of its genes that are parts of the chromosomes. Members in the initial set population are then grouped together and paired into parents.

\section{Step 2}

The parent chromosomes are then mated to generate a new set of offspring chromosomes. This mating procedure is also called crossover. Crossover is the procedure of splitting two chromosomes at some points in their string length and switching with the other chromosome to create two new chromosomes, each consisting of parts of the parent chromosomes. The split point does not have to be in the middle. For example, if $m=3$, and two parent strings are 000000 and 111111 , a mating might produce two strings: 001111 and 110000. This is illustrated in Figure 1.

\section{Step 3}

The individual bits of the offspring gene strings are then changed with small probability corresponding to mutation. Mutation is the random changing of one or more bits in a chromosome. It is useful for creating new genes that are not in the initial set population, or those that have evolved out of the population, but now would be beneficial (Ladd, 1996; Collier, 2000). For example, the two offspring might become 001101 and 110100. This is illustrated in Figure 2.

\section{Step 4}

The objective function is defined as channel utilisation in Equation (2), with the constraints defined in Equation (1). The objective function is channel utilisation with the constraints. We evaluate each of these strings including parents and offspring with the objective function via simulation, and we choose new parents probabilistically among the strings according to the fitness. The probability we chose is proportional to its fitness as follows:

$$
p_{i}=\frac{U_{i}}{\sum_{j} U_{i}} .
$$


Figure 1 Example of mating/crossover

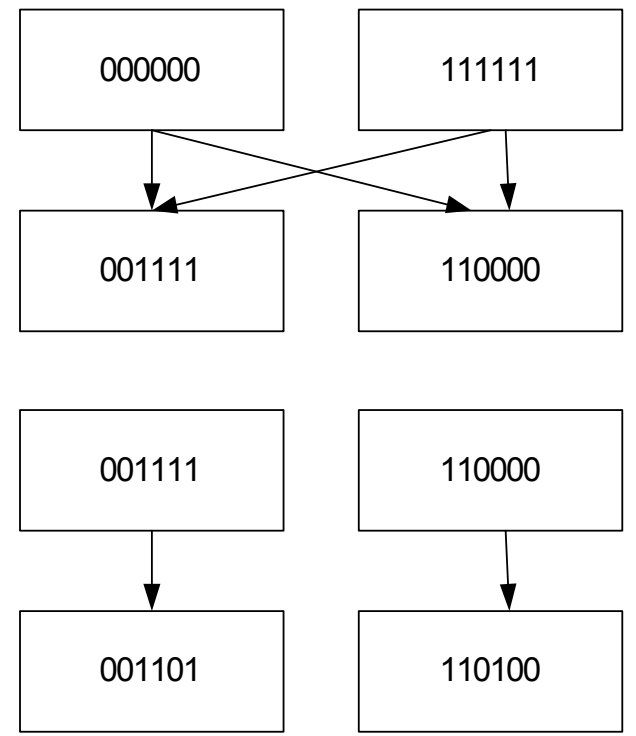

Figure 2 Examples of mutation

We assume that all the strings satisfy the constraints. Otherwise, we can let the probability be zero.

For those that have not been chosen, we will delete them with probability $1-p_{i}$. We call the chosen ones those that have not been deleted in the population. We will keep the size of the population constant by stopping to choose or stopping to delete when some threshold is reached. The population are then grouped together and paired into parents.

Step 5

If it has been more than $N$ iterations without improvement, go to Step 6. Otherwise, go to Step 2. $N$ is a pre-defined constant. Improvement is defined by comparing the average fitness with that of the previous generation.

Step 6

We choose the best fitful decision in terms of Equation (12) among all the members in the population.

The above algorithm can run online or offline. Note that the above algorithm just finds one near-optimal decision string for one system state. If this algorithm is running online, when the system state changes, the algorithm needs to run again to find the near-optimal decision string at the new system state. However, we can run this algorithm offline to find all near-optimal decision strings for all system states. 


\section{Simulation results}

In this section, we compare our genetic algorithm with the SMDP approach. Simulation parameters are illustrated in Table 1 . We assume that the handoff arrival rate is proportional to the new arrival rate, $\lambda_{i, h}=\alpha \lambda_{i, n}$. We let $\alpha=0.5$, that is $\lambda_{i, h}=0.5 \lambda_{i, n}$. $N$ is the number of iterations.

Table 1 Simulation parameters

\begin{tabular}{lc}
\hline Experimental parameters & Value \\
\hline $\mathrm{N}$ & 150 \\
$\mathrm{~m}$ & 2 \\
$\mathrm{~b}_{1}$ & 1 \\
$\mathrm{~b}_{2}$ & 2 \\
$\mathrm{D}_{1, \mathrm{HB}}$ & 0.01 \\
$\mathrm{D}_{2, \mathrm{HB}}$ & 0.02 \\
$\mathrm{C}$ & 25 \\
$1 / \mu_{1}$ & $500 \mathrm{sec}$. \\
$1 / \mu_{2}$ & $500 \mathrm{sec}$. \\
$\lambda_{1, \mathrm{~h}} / \lambda_{1, \mathrm{n}}$ & 0.5 \\
$\lambda_{2, \mathrm{~h}} / \lambda_{2, \mathrm{n}}$ & 0.5 \\
$1 / \mathrm{h}$ & $100 \mathrm{sec}$. \\
\hline
\end{tabular}

Figure 3 compares the channel utilisation for the SMDP approach and the genetic algorithm approach as Erlang load increases. Erlang load is defined as $\lambda_{i, r} \mu_{i}$. It is shown that the genetic algorithm approach's channel utilisation is very close to the optimal channel utilisation produced by the SMDP approach. The results reveal that the genetic algorithm approximates the optimal solution very well.

Figure 3 Channel utilisation versus Erlang Load

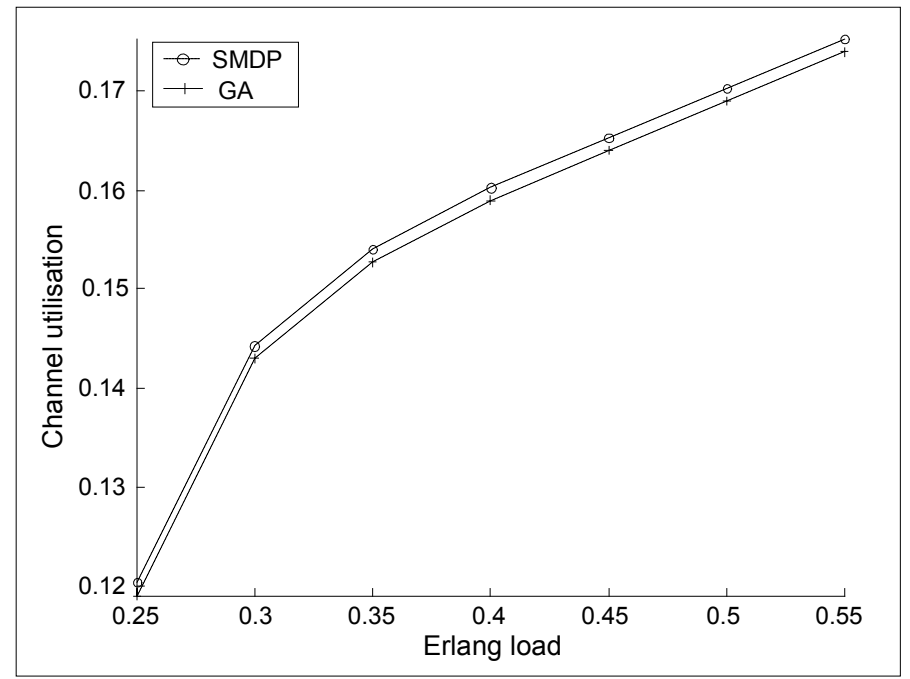


Figure 4 and Figure 5 show handoff dropping probabilities for class-1 and class-2, respectively. We observe that handoff dropping probabilities are bounded for both schemes and for both classes. In other words, QoS requirements are satisfied.

Figure 4 Handoff dropping probability for class-1

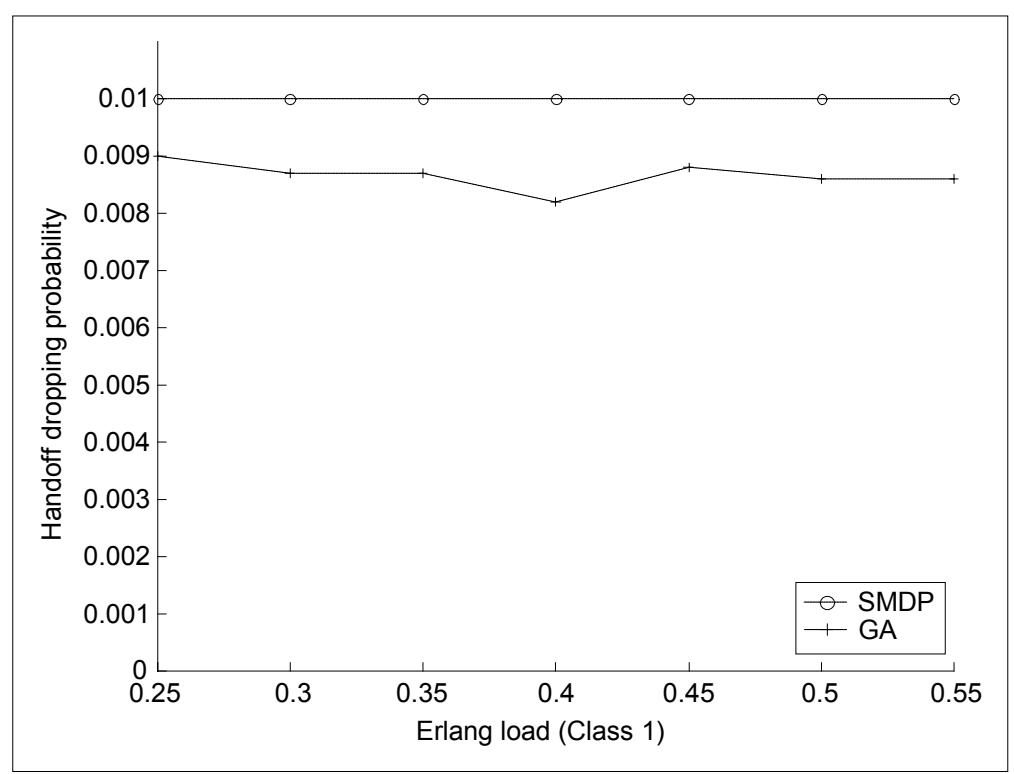

Figure 5 Handoff dropping probability for class-2

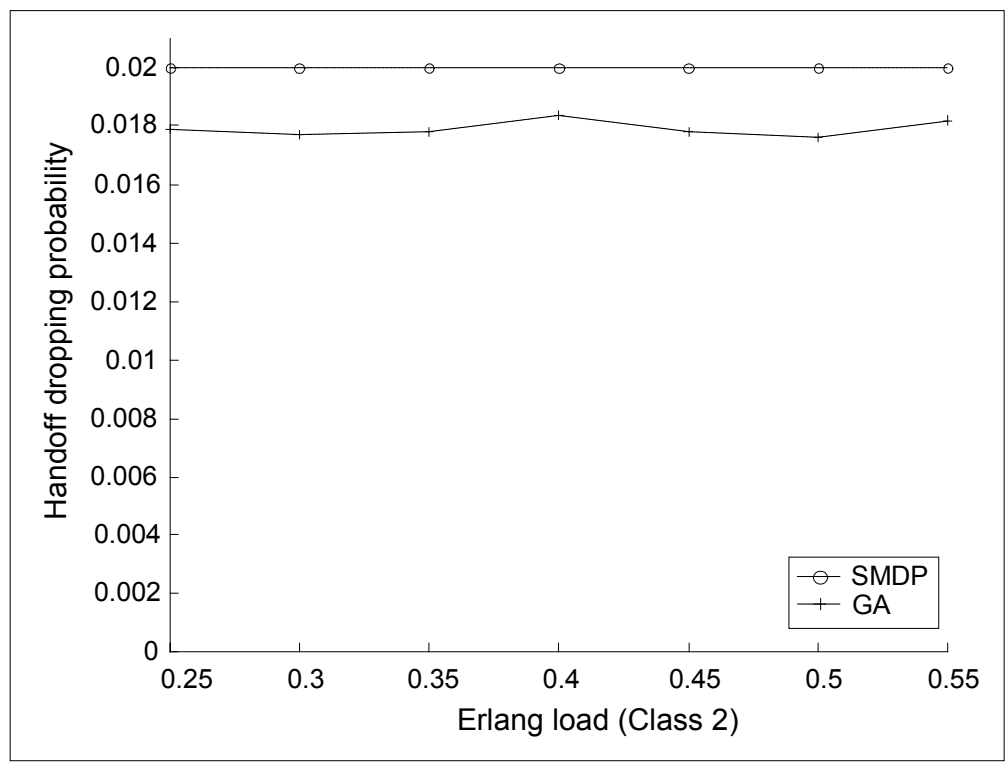




\section{Conclusion}

The Semi-Markov Decision Process (SMDP) approach can be used in CAC to provide an optimal solution. However, such a method fails when the state space and the action space are too large. The action space increases exponentially with $m$, and the state space is extremely large for the large $C$.

In this paper, we apply a genetic algorithm approach to address such problems as when the SMDP approach fails for multi-class multimedia services. This method overcomes the computational limits of the SMDP approach. We present the coding method and the genetic algorithm itself. Simulation results from the genetic algorithm are compared with the optimal solution obtained from linear programming for the SMDP approach. The results reveal that the genetic algorithm approximates the optimal solution very well, and at the same time QoS requirements are satisfied.

\section{References}

Alwan, A. (1996) 'Adaptive mobile multimedia networks', IEEE Communications Magazine, Vol. 34, No. 4, pp.34-51.

Ayyagari, D. and Ephremides, A. (1999) 'Admission control with priorities: approaches for multi-rate wireless systems', Mobile Networks and Applications, Vol. 4, pp.209-218.

Biaz, S. and Ji, Y. (2005) 'A survey and comparison on localisation algorithms for wireless ad hoc networks', International Journal of Mobile Communications, Vol. 3, No. 4, pp.374-410.

Biswas, S. and Sengupta, B. (1997) 'Call admissibility for multirate traffic in wireless ATM networks', Proceedings of IEEE INFOCOM'97, pp.650-659.

Choi, J., Kwon, T., Choi, Y. and Naghshineh, M. (2000) 'Call admission control for multimedia service in mobile cellular networks: a Markov decision approach', Proceedings of IEEE ISCC'00.

Collier, J.A. (2000) 'Genetic scheduling of batch processing in a multiple machine complex', Master Thesis, Wright State University.

Ho, C. and Lea, C. (1999) 'Improving call admission policies in wireless networks', Wireless Networks, Vol. 5, pp.257-265.

Karthik, S., Senthilkumar, V., Chidambararajan, B. and Srivatsa, S.K. (2004) 'Performance of TCP over satellite networks under severe cross-traffic using GA', International Journal of Mobile Communications, Vol. 2, No. 4, pp.382-394.

Kwon, T., Choi, Y. and Naghshineh, M. (1998) 'Optimal distributed call admission control for multimedia service in mobile cellular network', Proceedings of Mobile Multimedia Conference (MoMuc'98).

Kwon, T., Choi, Y., Bisdikian, C. and Naghshineh, M. (1998) 'Call admission control for adaptive multimedia in wireless/mobile network', Proceedings of ACM Workshop on Wireless Mobile Multimedia (WOWMOM'98), pp.111-116.

Kwon, T., Choi, Y., Bisdikian, C. and Naghshineh, M. (2003) 'QoS provisioning for adaptive multimedia in wireless/mobile networks', Wireless Network, Vol. 9, No. 1, pp.51-59.

Kwon, T., Park, I., Choi, Y. and Das, S. (1999) 'Bandwidth adaptation algorithms with multi-objectives for adaptive multimedia services in wireless/mobile networks', Proceedings of ACM Workshop on Wireless Mobile Multimedia_(WOWMOM'99), pp.51-58.

Ladd, S.R. (1996) Genetic Algorithms in C++, New York: M\&T Books.

Lou, Y., Pan, Y., Li, J., Xiao, Y. and Lin, X. (2004) 'A simulation study of overflow replacement policies for location management in mobile networks', International Journal of Mobile Communications, Vol. 2, No. 2, pp.103-127. 
Naghshineh, M. and Willebeek-LeMair, W. (1997) 'End-to-end QoS provisioning in multimedia wireless/mobile networks using a adaptive framework', IEEE Communications Magazine, Vol. 35, No. 11, pp.72-81.

Ramjee, R., Towsley, D. and Nagarajan, R. (1997) 'On optimal call admission control in cellular networks', Wireless Networks, Vol. 3, pp.29-41.

Rose, C. and Yate, R.D. (1996) 'Genetic algorithms and call admission to telecommunications networks', Computers and Operations Research, Vol. 23, No. 5, pp.485-499.

Tijms, H.C. (1986) Stochastic Modeling and Analysis: A Computational Approach, New York: Wiley.

Wang, S. and Wang, H. (2005) 'A location-based business service model for mobile commerce', International Journal of Mobile Communications, Vol. 3, No. 4, pp.339-349.

Wee, J. and Gutierrez, J.A. (2005) 'A framework for effective quality of service over wireless networks', International Journal of Mobile Communications, Vol. 3, No. 2, pp.138-149.

Xiao, Y., Chen, C.L.P. and Wang, B. (2002) 'Bandwidth degradation QoS provisioning for adaptive multimedia in wireless/mobile networks', Computer Communications, Elsevier Science, Vol. 25, No. 13, pp.1153-1161.

Xiao, Y., Chen, C.L.P. and Wang, Y. (2001) 'Optimal admission control for multi-class of wireless adaptive multimedia services', IEICE Transactions on Communications, Special Issue on Mobile Multimedia Communications, Vol. E84-B, No. 4, pp.795-804.

Xiao, Y., Li, H., Chen, C.L.P., Wang, B. and Pan, Y. (2005) 'Proportional degradation services in wireless/mobile adaptive multimedia networks', Wireless Communications and Mobile Computing, Vol. 5, No. 2, pp.219-243.

Yener, A. and Rose, C. (1997) 'Genetic algorithm applied to cellular call admission problem: local policies', IEEE Transaction on Vehicular Technology, Vol. 46, No. 1.

Yoon, D.I. and Lee, B. (1999) 'A distributed dynamic call admission control that supports mobility of wireless multimedia users', Proceedings of ICC'99, pp.1442-1446.

Zhang, Y. (1995) Solving Large-scale Linear Programming by Interior-point Methods under the MATLAB Environment, Department of Mathematics and Statistics, University of Maryland, Technical Report TR96-01.

Zhao, G.Y. (1999) 'Interior-point methods with decomposition for solving linear programs', Journal of Optimization Theory and Applications, Vol. 102, No. 1, pp.169-192. 